\title{
DIAGNÓSTICO DE ENFERMAGEM EM MULHERES SUBMETIDAS À REVASCULARIZAÇÃO DO MIOCÁRDIO
}

\author{
Paloma Cesar de Sales \\ Doutoranda em Enfermagem / New York University - USA \\ pcs325@nyu.edu \\ Simone Maria Muniz Bezerra da Silva \\ Doutora em Ciencias da Fisiologia / Universidade de Sao Paulo - Brasil \\ simonemunizm2@gmail.com \\ Fernanda Apolonio Rocha \\ Bacharel em Enfermagem / Universidade Estadual de Pernambuco - Brasil \\ fernanda.apolonio@hotmail.com
}

\section{RESUMO}

O número de pacientes do sexo feminino submetidas à Revascularização do Miocárdio (RVM) tem aumentado. Conhecida popularmente como ponte de safena, a RVM é o tipo mais comum de cirurgia cardíaca reconstrutora. $\mathrm{O}$ estudo teve por objetivos traçar o perfil epidemiológico das mulheres submetidas a este tipo de cirurgia em um hospital de referência em cardiologia em Recife-PE; bem como identificar os principais diagnósticos de enfermagem nestas pacientes. Identificamos que $83 \%$ das mulheres não praticavam atividades físicas antes da cirurgia e os principais diagnósticos de enfermagem encontrados foram: dor aguda e risco de infecção, ambos relacionados ao procedimento cirúrgico. Não menos importantes também foram identificados os diagnósticos de enfermagem padrão respiratório ineficaz, insônia e ansiedade com frequências de $60 \%, 50 \%$ e $43 \%$ respectivamente. O diagnóstico de enfermagem trata-se de uma das principais etapas que garantem a assistência de enfermagem eficiente e deve ser explorada no âmbito da assistência cardiovascular.

Palavras-chaves: Diagnósticos de enfermagem; Doenças cardiovasculares; Enfermagem eardiovascular.

\begin{abstract}
The number of patients were female, underwent myocardial revascularization have increased. Popularly known as bypass surgery, the myocardial revascularization (CABG) is the most common type of reconstructive heart surgery. The study aimed to delineate the epidemiological profile of women undergoing this type of surgery in a referral hospital for cardiology in Recife-PE, as well as identify key nursing diagnoses in these patients. We found that $83 \%$ of women did not practice physical activity before surgery and major nursing diagnoses found were acute pain and risk of infection related to the surgical procedure. Not least important, were also identified inefficient respiratory patter, insomnia and anxiety showing $60 \%$, $50 \%$ and $43 \%$ frequencies respectively. Nursing diagnosis it is one of the main steps that ensure efficient nursing care and should be explored in the context of cardiovascular care.
\end{abstract}

Keyword: Nursing diagnosis; Cardiovascular diseases; Cardiovascular nursing.

Persp. online: biol. \& saúde, Campos dos Goytacazes, 20 (6), 45-53, 2016

seer.perspectivasonline.com.br 


\section{INTRODUÇÃO}

A doença da artéria coronária (DAC) é o tipo mais prevalente da doença cardiovascular (ASSIS, STIPP, LEITE \& CUNHA, 2009). A mesma possui um espectro clínico importante que deve ser reconhecido adequadamente. Uma lesão pequena e não importante pode progredir, gradualmente, até limitar o fluxo sanguíneo e promover angina; uma simples lesão pode acumular, progressivamente, lipídios, plaquetas e fina capa de fibrose; pode promover inibição da síntese de colágeno pelos linfócitos $\mathrm{T}$ e aumento da degradação do colágeno, culminando com a ruptura da placa que, por sua vez, pode levar à formação do trombo, manifestada de diversas maneiras (COKKINOS 2008; GATTI et al, 2008). As doenças cardiovasculares representam um importante problema de saúde pública não só no Brasil, mas em todo o mundo, visto que constituem a principal causa de morbimortalidade e representam os mais altos custos em assistência médica. $\mathrm{O}$ universo de pacientes que apresenta DAC vem aumentando progressivamente em todo o mundo, uma vez que eles vivem mais em decorrência do tratamento dos quadros agudos (SILVA et al; 2010).

Utilizando-se os mesmos cálculos para o Brasil, temos, pelo menos, 900.000 brasileiros com angina do peito e cerca de 18.000 novos casos da doença ao ano, baseados num total de 30 casos de angina estável para cada caso de infarto agudo hospitalizado em um ano (MINISTÉRIO DA SAÚDE, 2010).

Os principais fatores de risco associados à DAC dividem-se em modificáveis (obesidade, tabagismo, diabetes mellitus, hipertensão arterial e dislipidemia) e não modificáveis (idade, sexo masculino e história familiar). A estratificação do risco coronariano na população geral depende da verificação desses elementos, associada aos achados clínicos, de forma que a intervenção efetiva nos fatores modificáveis altera o risco de um evento coronariano primário ou secundário (MINISTÉRIO DA SAÚDE, 2010).

Segundo Estatísticas de Mortalidade, no Brasil, as doenças cardiovasculares constituem importante causa de mortalidade, representando a principal causa de mortalidade por todas as causas. Estudos relatam que elas assumiram um papel preponderante desde os anos 60 nas capitais brasileiras e, em cidades como São Paulo, elas são a principal causa de morte desde o final dos anos 40. A comparação internacional das taxas de mortalidade dos principais centros do Brasil com a de outros países já mostrou valores elevados de mortalidade geral e para todo o conjunto das doenças cardiovasculares (MINISTÉRIO DA SAÚDE, 2010; SOUZA et al; 2011).

No entanto, ainda hoje, guarda-se a ideia amplamente aceita de que o infarto é uma condição exclusiva do homem de meia-idade, simplesmente pela sua maior prevalência no sexo masculino, na falsa suposição de que as mulheres estão, de certa forma, protegidas da doença. No entanto, mulheres com algum tipo de doença cardiovascular apresentam frequentemente maior número de fatores de risco, de condições comórbidas associadas e grande limitação funcional, resultando em uma população de alto risco de mortalidade, quando a doença se torna clinicamente manifesta (SOUZA et al., 2011). O aumento do índice de mulheres infartadas pode ser explicado pelo fato de que a menopausa pode ser um fator causal, pois $o$ infarto incide especialmente em mulheres a partir dos 50 anos.

Uma das explicações para isso é que, após a menopausa, as mulheres apresentam uma queda de estrógeno, hormônio feminino que, entre outras funções, promove elevados teores de lipoproteínas de alta densidade (HDL), apelidadas de 'bom colesterol', responsáveis por manter as artérias livres da arteriosclerose. Apesar de o risco cardíaco aumentar de duas a três vezes após a menopausa, o estilo de vida da mulher antes dessa fase pode, também, levar ao infarto. $\mathrm{O}$ ingresso da mulher no mercado de trabalho contribuiu em muito para isso. Além de exercer sua atividade profissional, a mulher tem outra jornada de trabalho: ser dona-de-casa. A mulher também adquiriu hábitos ruins da vida moderna, como o tabagismo e a má alimentação. Tais mudanças no comportamento feminino nos dias de hoje, embora tenha trazido muitas

Persp. online: biol. \& saúde, Campos dos Goytacazes, 20 (6), 45-53, 2016 seer.perspectivasonline.com.br 
conquistas como novos espaços profissionais e melhores salários, trouxeram também agravos à saúde que, até então, não eram encontrados entre as mulheres.

Sendo assim, o número de pacientes, do sexo feminino, submetidas à Revascularização do Miocárdio tem aumentado. Conhecida popularmente como ponte de safena, a Revascularização do Miocárdio (RVM) é o tipo mais comum de cirurgia cardíaca reconstrutora. $\mathrm{O}$ objetivo dessa cirurgia é aliviar a angina e preservar a função do miocárdio. Estudos mostraram que $90 \%$ dos pacientes pós-revascularização demonstram melhora da função cardíaca, com redução da necessidade de utilização de betabloqueadores e nitratos e que $60 \%$ apresentam eliminação de episódios anginosos, resultando em melhoria da qualidade de vida (MASCARENHAS et al; 2011).

A enfermagem enquanto ciência é organizada por sistemas. Portanto, o diagnóstico de enfermagem como parte deste sistema é uma descritiva sobre o estado de saúde de um cliente (que pode ser um indivíduo, uma família, ou uma comunidade em geral). A função do diagnóstico de enfermagem é facilitar o cuidado da enfermagem. As análises devem ser feitas a partir do estado de saúde dos clientes envolvidos, sendo que o enfermeiro cria sua opinião e assim contribui para evolução do mesmo. Vale ressaltar que o diagnóstico de enfermagem é desvinculado do diagnóstico médico, todavia este pode auxiliar no estabelecimento de um diagnóstico de enfermagem, ou não ter correlação.

Existem diversas organizações que regulam e definem diagnósticos de enfermagem no mundo. $\mathrm{Na}$ América do Norte o principal delas é a NANDA. Outras organizações incluem a ACENDIO na Europa, a AFEDI (de língua francesa) e a AENTDE (língua castelhana) (CARPENITO, 2008).

Portanto o objetivo do diagnóstico de enfermagem é padronizar a terminologia utilizada por enfermeiros, tais como descrições de problemas de enfermagem, intervenções e resultados. Proponentes do uso de diagnósticos de Enfermagem argumentam que o uso destes diagnósticos tornam o processo de cuidado mais científico e baseado em evidência.

Diante desse cenário, este estudo teve por objetivos traçar o perfil epidemiológico das mulheres submetidas à Revascularização do Miocárdio e identificar os principais diagnósticos de enfermagem nestas pacientes.

\section{METODOLOGIA}

Trata-se de uma pesquisa descritivo-exploratória, do tipo transversal, com abordagem quantitativa. A pesquisa descritiva dá-se através da observação características de um determinado fenômeno, procurando descrevê-los, classificá-los e interpretá-los e, através de parâmetros estatísticos, o método quantitativo o quantifica e analisa. Sendo do tipo transversal por que os dados são coletados em um único instante do tempo, obtendo assim, um recorte de informações momentâneas (LAKATOS \& MARCONI, 2007).

A pesquisa foi realizada no Pronto Socorro Cardiológico de Pernambuco (PROCAPE), na Cidade do Recife, no bairro de Santo Amaro. Os campos selecionados para a pesquisa foram a unidade de recuperação cardiotorácica e as unidades coronarianas I e II, bem como as enfermarias coronarianas. A escolha deste hospital como local para o estudo se deu por ser um Hospital Escola de referência em cardiologia no Estado de Pernambuco.

Os sujeitos do estudo foram 30 pacientes do sexo feminino submetidas à cirurgia de revascularização do Miocárdio no período de coleta de dados, durante os meses de outubro à novembro de 2011. Foi utilizado um instrumento semiestruturado, do tipo check-list e a observação sistemática para o levantamento dos

Persp. online: biol. \& saúde, Campos dos Goytacazes, 20 (6), 45-53, 2016

seer.perspectivasonline.com.br 
principais diagnósticos de enfermagem segundo a taxonomia da North American Nursing. Diagnosis Association - NANDA 2012.

Quanto a critérios de inclusão e exclusão, foram incluídas na amostra apenas mulheres submetidas a cirurgia de revascularização do miocárdio durante o período de coleta dos dados, e que concordaram em participar do estudo, mediante assinatura do Termo Livre e Esclarecido - TCLE. Foram excluídas mulheres que apresentaram dificuldades de fala ou escrita para participar da pesquisa.

Este estudo teve a aprovação do Comitê de Ética do Hospital Universitário Oswaldo Cruz, mediante protocolo: CAAE: 0148.0.097.000-11. Os dados serão apresentados em forma de gráficos e tabelas e foram analisados através do programa estatístico Epi Info 7.0.

\section{RESULTADOS}

Em relação ao perfil sóciodemográfico das mulheres submetidas à revascularização do miocárdio, 90\% das entrevistadas possuíam idade acima de 51 anos, conforme tabela 1.

Tabela 1. Perfil das mulheres submetidas à vascularização do miocárdio de acordo com a faixa etária. Recife, 2012.

\begin{tabular}{|c|c|c|}
\hline IDADE & QTD & $\%$ \\
\hline $41-50$ ANOS & 3 & $10 \%$ \\
\hline $51-60$ ANOS & 15 & $50 \%$ \\
\hline MAIS DE 61 ANOS & 12 & $40 \%$ \\
\hline Total geral & 30 & $100 \%$ \\
\hline
\end{tabular}

No que se refere à idade associada à renda familiar, identificamos que a maioria das mulheres entrevistadas possui renda familiar de até dois salários mínimos considerando os valores vigentes (n. 10) correspondendo a $33 \%$ do total da amostra.

Tabela 2. Perfil de mulheres submetidas à revascularização do miocárdio em relação à idade associada com a renda familiar, Recife -2012 .

\begin{tabular}{cccc}
\cline { 2 - 4 } Idade & \multicolumn{3}{c}{ Renda Familiar } \\
Entre 3 à 5 Salários & Até 2 Salários & Total \\
\hline 41 50 ANOS & $3 \%$ (n. 1) & $7 \%($ n.2) & $10 \%$ \\
\hline MAIS DE 61 ANOS & $17 \%($ n.5) & $33 \%$ (n.10) & $50 \%$ \\
\hline Total & $17 \%($ n.5) & $23 \%$ (n.7) & $40 \%$ \\
\hline
\end{tabular}

Persp. online: biol. \& saúde, Campos dos Goytacazes, 20 (6), 45-53, 2016 seer.perspectivasonline.com.br 
Em relação à prática de atividades físicas antes da doença cardiovascular, $83 \%$ das entrevistadas informaram que nunca praticaram atividade física e dentre as mulheres que praticavam atividade física $14 \%$ informaram que realizavam a atividade "às vezes" (até duas vezes por semana) e 3\% praticavam "sempre' (mais de duas vezes por semana), conforme gráfico à seguir.

Figura 1. Frequência das atividades físicas antes da doença cardiovascular entre as mulheres submetidas à revascularização do miocárdio, Recife - 2012.

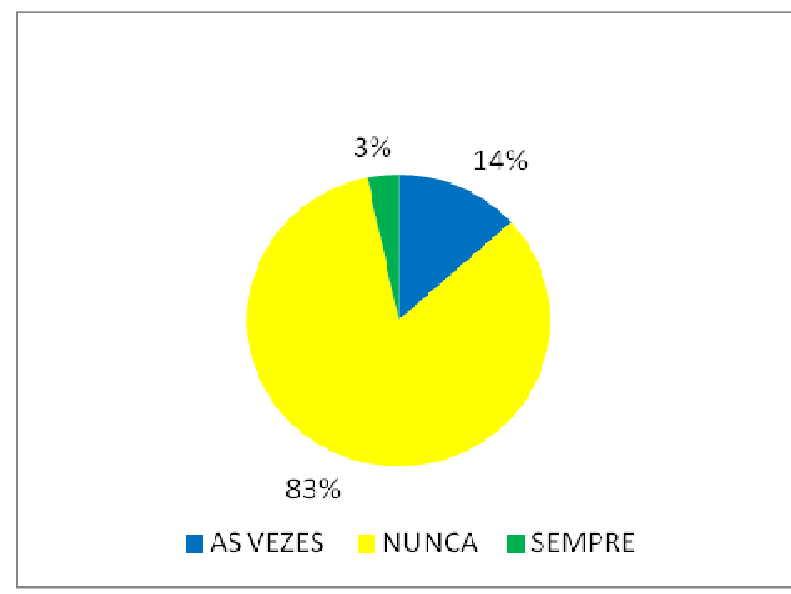

Em relação aos principais diagnósticos de enfermagem levantados no momento da coleta de dados, utilizamos como modelo a taxonomia da North American Nursing. Diagnosis Association- NANDA e todas as pacientes obtiveram diagnóstico de risco para infecção e dor aguda associadas ao procedimento cirurgico.

Figura 2. Principais diagnósticos de Enfermagem associados as mulheres submetidas à revascularização do miocárdio, Recife-2012.

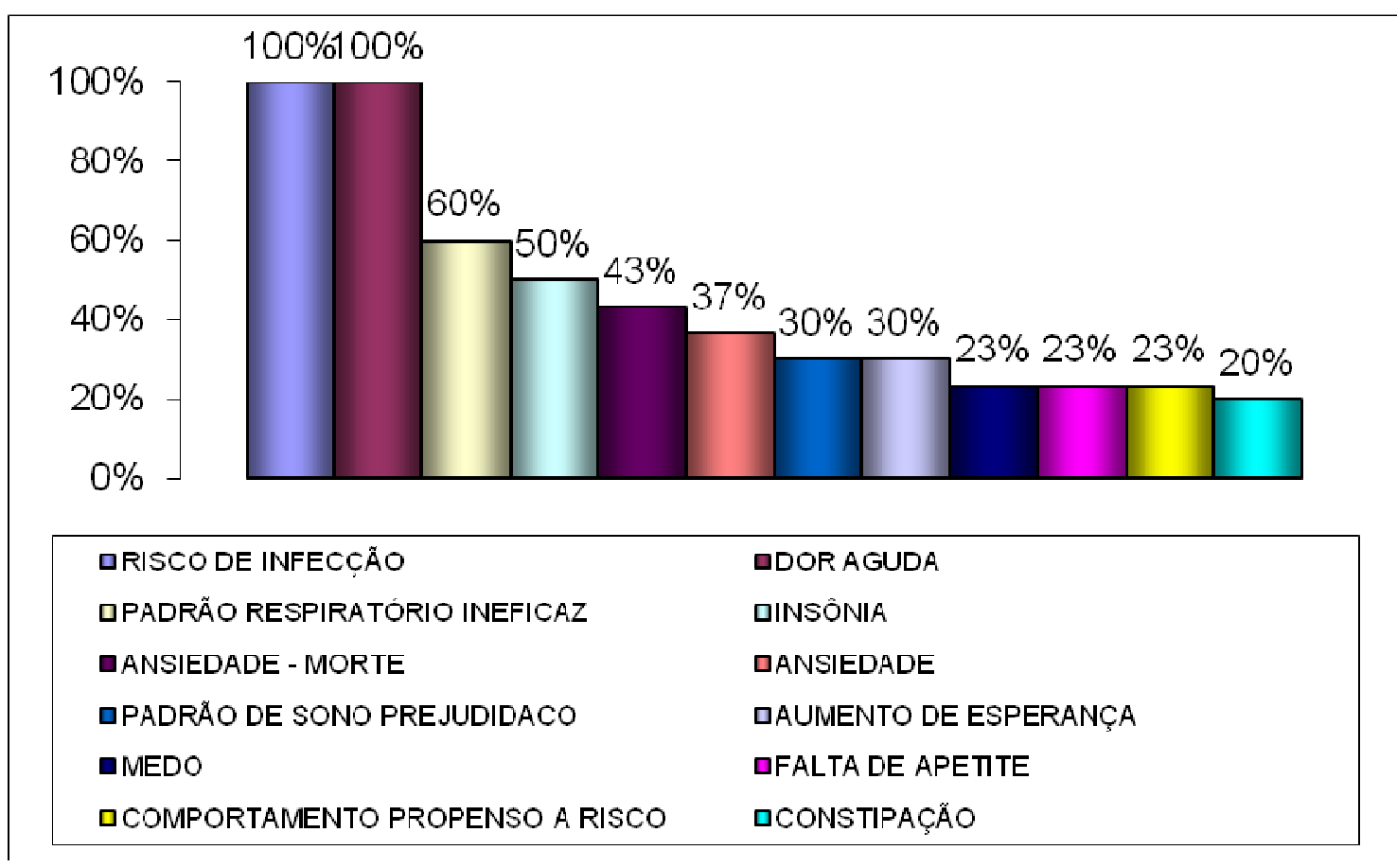

Persp. online: biol. \& saúde, Campos dos Goytacazes, 20 (6), 45-53, 2016 seer.perspectivasonline.com.br 


\section{DISCUSSÃO}

A cirurgia de Revascularização do Miocárdio consiste em um enxerto arterial coronário usando a veia safena autógena e tem como objetivo isolar o vaso obstruído e, assim, restabelecer a perfusão da artéria coronária. Esse tipo de cirurgia, cuja maior vantagem é a durabilidade, tem a finalidade de preservar o miocárdio (CARVALHO et al; 2008; TAKAHASHI, 2008) Suas indicações predominantes são para pacientes sintomáticos e que tenham intolerância ao tratamento medicamentoso, nos casos de lesões importantes impossíveis de ser corrigidas com angioplastia, em infarto agudo do miocárdio (IAM), como prevenção ou correção ou por choque cardiogênico, no tratamento das complicações do infarto agudo do miocárdio e na angina instável e piora do quadro clínico (BARROS, 2009).

Devido ao fato de que a enfermagem é responsável pelo cuidado do paciente cirúrgico, é ela a grande responsável em orientar o paciente no pós-operatório. O pós-operatório é o período durante o qual ocorre a recuperação do paciente. Nele a assistência de enfermagem está relacionada com as intervenções destinadas a prevenir ou tratar complicações e proporcionar ao paciente o retorno às atividades do cotidiano. Essas intervenções são constituídas de um conjunto de etapas, como: coleta de dados, diagnóstico de enfermagem, planejamento, implementação e avaliação, nas quais sobressai a importância do cuidado individual mediante uma abordagem de solução de problemas fundamentados em teorias e modelos conceituais de enfermagem (BARROS, 2009).

No desenvolvimento do processo, a etapa do diagnóstico de enfermagem tem se destacado e estudos a este respeito têm sido aprofundados. Trata-se de uma etapa complexa, cuja sistemática envolve avaliação clínica das respostas do paciente ou grupos aos problemas de saúde, e fornece a base para a intervenção de enfermagem no intuito de se atingir os resultados esperados. Por meio do raciocínio clínico acerca de dados coletados via entrevista clínica e exame físico, podem ser identificados os diagnósticos classificados como de enfermagem (FIGUEIREIDO et al; 2006).

Neste estudo identificamos a dor aguda e o risco para a infecção como principais diagnósticos nas mulheres submetidas à vascularização do miocárdio em função do próprio procedimento cirúrgico oferecer uma exposição maior a infecção por bactérias no nível sistêmico do paciente. A partir da identificação deste diagnóstico risco, as ações de enfermagem devem ser planejadas e definidas com a finalidade da redução do risco para o paciente, com medidas de controle e técnicas assépticas no cuidado pós-operatório.

Em relação ao diagnóstico de dor aguda, este se faz muito importante para o trabalho da enfermagem, pois vem sendo recentemente considerada na comunidade científica como o $5^{\circ}$ sinal vital (NASCIMENTO \& KRELING, 2011). E é caracterizada por ser uma experiência subjetiva, a dor não pode ser objetivamente determinada por instrumentos físicos que, usualmente, mensuram o peso corporal, a temperatura, a altura, a pressão sanguínea e o pulso. Em outras palavras, não existe um instrumento padrão que permita a um observador externo, objetivamente, mensurar essa experiência interna, complexa e pessoal.

Não menos importantes foram identificados os diagnósticos de enfermagem padrão respiratório ineficaz, insônia e ansiedade com frequência de 60\%, 50\% e 43\% respectivamente. Estes diagnósticos são relevantes pois se coadunam entre si na experiência de internação vivida por estas mulheres. Ansiedade, stress e medo são relatados e conhecidos na ciência da Psicologia como manifestações comuns em pacientes internados (BOIZONAVE \& BARROS, 2003).

O estudo de Gascon et. al., (2012) revelou que quanto maior o período de internação dos pacientes em um hospital universitário, maior a era a frequência de sentimentos de ansiedade e depressão. Desta forma, entendemos que por se tratar de um ambiente hospitalar que foge a rotina do seio familiar, as mulheres submetidas a cirurgia de revascularização também apresentaram diagnósticos de ansiedade. 
O diagnóstico de padrão respiratório ineficaz, pode estar associado aos efeitos residuais da anestesia. O estudo de Souza, Carvalho e Paldino (2012) revelou que o diagnóstico de padrão respiratório ineficaz está associado ao efeito da anestesia na depressão do centro respiratório em pacientes em recuperação pósanestésica. Sendo assim, cuidados com a oxigeno-terapia tornam-se fundamental para a enfermagem atender as necessidades deste diagnostico especifico.

Por fim, o diagnóstico de insônia trata-se de uma manifestação comum em pacientes internados e pode ser desencadeado por diversos fatores emocionais e biológicos (SOUZA \& REIMAO, 2004). Desta forma, também pode estar associado as manifestações de ansiedade e padrão respiratório ineficaz. Cabe ao enfermeiro promover cuidados que favoreçam o sono dos pacientes como por exemplo, promover um ambiente tranquilo para o repouso, livre de ruídos que possam vir a aumentar ansiedade e consequentemente insônia no paciente.

Para o desenvolvimento das ações de enfermagem a formulação dos diagnósticos de enfermagem é o principal aspecto dentro do processo da sistematização da enfermagem o que por sua vez fortalece a cuidado da enfermagem cientificamente.

A ciência da enfermagem está baseada em uma ampla estrutura teórica e o processo de enfermagem é uma das ferramentas por meio da qual essa estrutura é aplicada à prática da enfermagem - ou seja - é o método de solução dos problemas do cliente. Sistematizar o cuidado é ainda algo novo para muitas realidades que encontramos nos serviços de saúde. A importância que o mesmo significa para a organização da assistência vai além da estrutura organizacional ${ }^{19}$.

O processo de enfermagem é entendido por Alfaro-LeFevre, como um sistema humanizado por se basear na crença de que à medida que planejamos ou proporcionamos cuidados, devemos considerar exclusivamente os interesses, os ideais e os desejos do cliente que necessita do atendimento de saúde e isso inclui o sujeito, sua família, e a comunidade em seu entorno (KOERICH et al; 2007).

Os diagnósticos de enfermagem para pacientes cardiovasculares também contribui para uma assistência de enfermagem no cuidado focado na prevenção e controle das doenças cardiovasculares tão prevalentes na população brasileira.

Concluímos este estudo acreditando que é fundamental o processo da sistematização da assistência de enfermagem principalmente no que se refere à formulação de diagnósticos voltados ao cuidado cardiovascular. A enfermagem possui vários modelos de processos para a assistência de enfermagem, nos quais o enfermeiro deve se apropriar e incorporar na sua prática assistencial aquela que vai de acordo com o seu contexto e necessidades no campo assistencial. Considero esse parágrafo discussão.

\section{CONCLUSÃO}

Na Conclusão deve destacar os principais resultados da caracterização dos pacientes e os principais DE encontrados. O processo de enfermagem é a ferramenta mais organizada e apesar de complexa, a implementação da sistematização da assistência da enfermagem deve ser planejada a fim de garantir que a inovação seja compatível com as necessidades tanto dos clientes quanto das metas da unidade de serviço, sendo fundamental um processo de avaliação contínua para que a sua introdução no ambiente possa ser sujeita a readaptações necessárias às características do cuidado.

Persp. online: biol. \& saúde, Campos dos Goytacazes, 20 (6), 45-53, 2016 seer.perspectivasonline.com.br 


\section{REFERÊNCIAS}

ASSIS, L.S; STIPP, M.A.C; LEITE, J.S; CUNHA, N.M. A atenção da enfermeira à saúde cardiovascular. Esc Anna Nery Rev Enferm 2009 abr-jun; n.13, Vol.2 p. 265-70.

BARROS, A.L.B.L. Classificações de diagnóstico e intervenção de enfermagem: Nanda-NIC. Acta Paul Enferm. 2009; vol. 22(Esp. 70 anos) pg.864-7.

BOIZONAVE, L. F; BARROS, M. T. Ansiedade e depressão: reações psicológicas em pacientes hospitalizados Aletheia, n. 17-18, Jan/Dec, 2003, p. 135-143.

CARPENITO, M.L.J. Nursing Diagnosis: Application to Clinical Practice. [S.1.: s.n.], 2008. p. 6-18 pp. ISBN 0-7817-8121-3

CARVALHO, A.R.S, MATSUDA, L.M, CARVALHO, M.S.S; ALMEIDA, R; SCHNEIDER, D; PICCOLI, M. Estudo retrospectivo das complicações intra-operatórias na cirurgia de revascularização do miocárdio. Rev. Eletr. Enf. [Internet]. 2008; vol.10, n.4 p.1057-65.

COKKINOS, D.V. Tratamento da Doença Arterial Coronariana; Simpósio Internacional sobre abordagens atuais para avaliação e tratamento de infarto do miocárdio e isquemia; 2008. Disponível em: http://www.cardios.com.br/ishne/infarto/palestras/coronariana-cokkinos.pdf Acesso em: 16/08/2012.

DOURADO, G.K.S; COELHO, M.J; Cuidados de enfermagem ao homem com doença arterial coronariana vítima de infarto agudo do miocárdio; $16^{\circ}$ Seminário Nacional de Pesquisa em Enfermagem; Junho 2011; vol.1, p. 900-902.

FIGUEIREDO, R.M; ZEM-MASCARENHAS, S.H; NAPOLEÃO, A.A; CAMARGO, A.B. Caracterização da produção do conhecimento sobre sistematização da assistência de enfermagem no Brasil. Rev Esc Enferm USP. 2006; n.40, vol.2, p.299-303.

GATTI, ET. AL; Avaliação dos fatores de risco para doença arterial coronariana em pacientes de São Caetano do Sul segundo o Escore de Framingham e sua relação com a síndrome metabólica; Arq Sanny Pesq Saúde n.1, vol.1, p.8-17, 2008.

GASCON, M. R. P., et al. Prevalence of depression and anxiety disorders in hospitalized patients at the dermatology clinical ward of a university hospital. Arq. Bras. Dermatol., Rio de Janeiro, v. 87, n. 3, p. 403407, Disponivel em: <http://www.scielo.br/scielo.php?script=sci_arttext\&pid=S036505962012000300008\&lng=en\&nrm=iso $>$. access on 17 Feb. 2016. http://dx.doi.org/10.1590/S036505962012000300008 .

KOERICH, M.S; BACKES, D.S; NASCIMENTO, K.C; ERDMANN, A.L. Sistematização da assistência: aproximando o saber acadêmico, o saber-fazer e o legislar em saúde. Acta Paul Enferm. 2007, n.20, vol.4, p.446-51.

LAKATOS, E.M; MARCONI, M.A. Fundamentos de metodologia científica. n.6. ed. São Paulo: Atlas, 2007.

MASCARENHAS, N.B; PEREIRA, A; SILVA, R.S, SILVA, M.G; Sistematização da Assistência de Enfermagem ao portador de Diabetes Mellitus e Insuficiência Renal Crônica; Rev. bras. enferm. vol.64 no.1 Brasília Jan./Feb. 2011

Persp. online: biol. \& saúde, Campos dos Goytacazes, 20 (6), 45-53, 2016 seer.perspectivasonline.com.br 
MINISTÉRIO DA SAÚDE ELSA Brasil: maior estudo epidemiológico da América Latina. Rev. Saúde Pública, São Paulo, 2009; (43):1.

MINISTÉRIO DA SAÚDE; Portal da Saúde - Sistema Único de Saúde; 2010. Disponível em: http://portal.saude.gov.br/portal/aplicacoes/noticias/default.cfm?pg=dspDetalheNoticia\&id_area=124\&CO_ NOTICIA=11994 Acesso em: 17/08/2012.

NASCIMENTO, LA; KRELING, M.G.D.K. Avaliação da dor como quinto sinal vital: opinião de profissionais de enfermagem Acta paul. enferm. São Paulo 2011; vol.24, p.1.

PEREIRA, J.M.V; ET. AL. Diagnósticos de enfermagem de pacientes hospitalizados com doenças cardiovasculares, Esc. Anna Nery; Out. Dez, 2011; vol.15, p.4.

SILVA, E.G.C; OLIVEIRA, V. C; NEVES, G.B.C; GUIMARÃES, T.M.R; O conhecimento do enfermeiro sobre a Sistematização da Assistência de Enfermagem: da teoria à prática; Rev Esc Enferm USP; 2011; n.45, vol.6, p. 1380-6.

SILVA, S.S; CARITÁ, E. C; MORAIS, E.R.E.D; fatores de risco para doença arterial coronariana em idosos: Análise por enfermeiros utilizando ferramenta computacional Esc Anna Nery; 2010; out-dez; n.14, vol.4, p.797-802.

SOUZA, T. M; CARVALHO, R; PALDINO, C. M. Resumos diagnósticos, prognósticos e intervenções de enfermagem na sala de recuperação pós-anestésica. Rev. SOBECC, São Paulo. Out./Dez 2012; n. 17, vol.4, p. 33-47.

SOUZA, J. C; REIMAO, R. Epidemiologia da Insônia. Psicologia em Estudo, Maringá, v. 9, n. 1, p. 3-7, 2004.

SOUZA, L.P.S; SOUSA, M.H.M; CARVALHO, I.Z; GRAVENA, A.A.F. Fatores de risco cardiovascular em mulheres na pós-menopausa; VII Encontro Nacional de Produção Científica, Out; 2011.

TAKAHASHI, A.A; BARROS, A.L.B.L; MICHEL, J.L.M; SOUZA, M.F; Dificuldades e facilidades apontadas por enfermeiras de um hospital de ensino na execução do processo de enfermagem. Acta Paul Enferm. 2008; n.21, vol.1, p.32-8.

Persp. online: biol. \& saúde, Campos dos Goytacazes, 20 (6), 45-53, 2016 seer.perspectivasonline.com.br 